\title{
Landscape genetics: the correlation of spatial and genetic distances of Oncomelania hupensis, the intermediate host snail of Schistosoma japonicum in mainland China
}

\author{
Shi-Zhu Li ${ }^{1}$, Yi-Xiu Wang, ${ }^{1,2}$ Kun Yang ${ }^{3}$, Qin Liu', Qiang Wang ${ }^{1}$, Yi Zhang ${ }^{1}$, Xiao-Hua \\ $\mathrm{Wu}^{1}$, Jia-Gang Guo ${ }^{1}$, Robert Bergquist ${ }^{4}$, Xiao-Nong Zhou ${ }^{1}$ \\ ${ }^{1}$ National Institute of Parasitic Diseases, Chinese Center for Disease Control and Prevention, Shanghai, \\ 200025, China; ${ }^{2}$ Life Science College, Shaanxi Normal University, Xian, 610062, China; Jiangsu \\ Institutes of Parasitic Diseases, Wuxi, 214064, China; ${ }^{4}$ Ingerod 407, Brastad, Sweden
}

\begin{abstract}
Two internal transcribed spacer (ITS1 and ITS2) sequences of the non-coding region of the rDNA and a 16S RNA gene fragment of the mtDNA of Oncomelania bupensis, the intermediate host snail of Schistosoma japonicum in mainland China, have been investigated with a view to illustrate the influence of the environment on genetic differentiation. Thirteen populations of O. hupensis snails, representing four types of ecological settings, were collected to compare genetic and spatial distances. The length of the ITS1-5.8S-ITS2 gene sequence showed a range from 752 to 796 bp with a guanine-cytosine (GC) content of $49.1-50.3 \%$, while that of the $16 \mathrm{~S}$ sequence ranged from 505 to $508 \mathrm{bp}$ with a GC content of $33.5-35.1 \%$. The $5.8 \mathrm{~S}$ fragment was shown to be highly conserved and it was therefore removed in the subsequent analysis. In contrast, the ITS flanking sequences and the $16 \mathrm{~S}$ fragment were found useful for further study as their degree of polymorphism amounted to $13.2 \%$ and $8.6 \%$, respectively. The genetic relationship was investigated using tools based on maximum parsimony, minimum evolution and neighbour-joining algorithms. Four branches of O. hupensis were found to be clearly represented on the $16 \mathrm{~S}$ phylogenetic tree, namely (i) the mountainous region population (from Sichuan and Yunnan provinces); (ii) the Karst region population (from Guangxi autonomous region); (iii) the population representing the region of swamps and lakes along the Yangtze River basin which stretches through the Anhui, Hunan, Hubei, Jiangxi, Jiangsu and Zhejiang provinces; and (iv) the littoral, hilly region population (from Fujian province). These four population branches were found to be correlated to environmental features based on the concept of landscape ecology attributing genetic differentiation to differences in ecological features. However, only three main branches could be found on the ITS1-ITS2 phylogenetic tree. The swamps and lakes population (from the Yangtze River basin) and the littoral, hilly population (from Fujian province) were clustered on the third branch in spite of these two populations not being spatially related, i.e. no firm genetic demarcation between their snail populations was found. Thus, it seems that the Fujian population does not constitute a separate branch but belongs to the third branch. This fact, together with the strong genetic evidence that the subspecies $O$. hupensis guangxiensis represents a discrete branch, support the hypothesis that genetic differentiation of O. hupensis in mainland China is ultimately structured by landscape ecology.
\end{abstract}

Keywords: Oncomelania hupensis, ITS1-5.8S-ITS2, 16S, genetic variation, subspecies, snail population, landscape genetics, China.

Corresponding author:

Xiao-Nong Zhou

National Institute of Parasitic Diseases

Chinese Center for Disease Control and Prevention

Shanghai 200025, People's Republic of China

Tel. +86 216473 8058; Fax + 862164332670

E-mail: ipdzhouxn@sh163.net

\section{Introduction}

Oncomelania hupensis is the sole intermediate snail host of Schistosoma japonicum, which therefore completely depends on this snail species for its transmission (Wang et al., 2009). 
The epidemiology and control of schistosomiasis continue to be a strong research focus due to the great importance of this disease in mainland China (Davis et al., 1995; Chitsulo et al., 2000; Zhou et al., 2005, Chen et al., 2007; Wang et al., 2008, 2009). This includes the development and application of molecular systematic techniques for the classification and population genetics of the snail (Hope et al., 1994; Spolsky et al., 1996; Xu and Zheng, 2003). As publicized in the National Center for Biotechnology Information (NCBI) database (http://www.ncbi.nlm.nih.gov/), Oncomelania is a recognized taxonomic group comprised of seven subspecies in the world, three of which are distributed in mainland China, namely O. hupensis hupensis, O. hupensis robertsoni and $O$. hupensis tangi (http://www.ncbi.nlm.nih.gov/Taxonomi/). However, there are uncertainties regarding O. hupensis tangi (Davis et al., 1997), while an additional recognized subspecies, O. hupensis guangxiensis, is now considered a discrete subspecies based on geographic distribution and amplified fragment length polymorphism (AFLP) (Zhou et al., 2007a,b).

From the spatial point of view, the areas where Oncomelania is mainly located in mainland China fall into four different ecological settings, i.e. the region of swamps and lakes in the Yangtze River basin (part of the Anhui, Hubei, Hunan, Jiangsu, Jiangxi and Zhejiang provinces), the mountainous region of the Sichuan and Yunnan provinces, the hilly, littoral part of the Fujian province, and the Karst landscape of Guangxi autonomous region (Chen et al., 2007; Zhou et al., 2007a). As the ecology varies considerably between these areas, it seems likely that features of the landscape have influenced and channeled the genetic variations of O. hupensis, into the different subspecies we see today, a situation not only demanding an understanding of ecology, but also a detailed knowledge of population genetics.

Landscape genetics is a relatively new discipline focusing on how the environment models populations and subspecies of organisms at the DNA level.
This approach combines the tools of molecular genetics with the principles of landscape ecology to spatially map allele frequencies and correlate genetic patterns with the limits set by the environment. The new concept promises to facilitate our comprehension of how geographical and environmental features structure the genetic variation at the population and speciation levels (Manel et al., 2003; Manel and Segelbacher, 2009). In spite of considerable amount of research on the population genetics of O. hupensis, the knowledge of the molecular genetics of this snail is still relatively limited. For example, only few sets of gene sequences have been deposited in GenBank (http://www.ncbi.nlm.nih.gov), most of them focused on the mitochondrial sequences of the cytochrome oxidase subunit 1 (COI) (Wilke et al., 2000, 2006; Shi et al., 2002).

This study aimed to amalgamate spatial statistics, geographical information system (GIS) and informative molecular markers with ecological biogeography and molecular ecology to better understand population biology and the evolution of O. hupensis. It is based on the supposition that the four types of environment mentioned above have channeled the genetics of this snail through environmental features resulting in four different ecotypes. To investigate this from the population genetics perspective, we have sequenced part of the O. hupensis mitochondrial $16 \mathrm{~S}$ gene, as well as the nuclear 5.8S rRNA gene with its two internal transcribed spacer (ITS) fragments (ITS1 and ITS2). The latter, flanking sequences emanate from a non-coding rDNA region and are characterized by a relatively fast evolutionary rate (Stothard et al., 1996), which facilitated the exploration of the genetic variation and phylogeny of this snail species.

\section{Materials and methods}

\section{Snail sampling}

Snails of the O. hupensis species were collected from 13 sites in 10 provinces in mainland China, representing four different landscape ecology populations, making up a total of 30 different isolates, 
which incorporated the three subspecies publicized in GenBank. We also included an O. hupensis quadrasi isolate (coded as Quadrasi) from the Philippines in the study as an out-group for the planned phylogeny analysis. The geographical origins and locations of all isolates investigated are shown in Table 1 and Figure 1.

\section{DNA preparation}

After removal of the gut and digestive glands from the soft parts of the snails, the muscle tissues from each single isolate were digested for 3 hours at $56^{\circ} \mathrm{C}$ in water bathing with proteinase $\mathrm{K}$ (Amresco Inc. Solon, OH, USA) followed by the standard DNA

Table 1. Information regarding the collected samples of Oncomelania hupensis.

\begin{tabular}{|c|c|c|c|c|}
\hline Population & Site, province, country & Site code & Date & Coordinates \\
\hline Swamps and lakes region & Tongling, Anhui, China & AHTL & $5 / 12 / 2007$ & $29.94904 \mathrm{~N} ; 118.45947 \mathrm{E}$ \\
\hline \multirow[t]{6}{*}{$\left(\mathrm{n}_{\text {ITS1-ITS2 }}=18, \mathrm{n}_{16 \mathrm{~S}}=15\right)$} & Yueyang, Hunan, China & HNYY & $3 / 28 / 2007$ & $29.08614 \mathrm{~N} ; 113.05955 \mathrm{E}$ \\
\hline & Hanshou, Hunan, China & HNHS & $4 / 14 / 2007$ & $28.92238 \mathrm{~N} ; 112.15158 \mathrm{E}$ \\
\hline & Yangxin, Hubei, China & HBYX & $3 / 29 / 2007$ & $29.82804 \mathrm{~N} ; 115.23305 \mathrm{E}$ \\
\hline & Yugan, Jiangxi, China & JXYG & $4 / 14 / 2007$ & $28.79958 \mathrm{~N} ; 116.34376 \mathrm{E}$ \\
\hline & Yangzhou, Jiangsu, China & JSYZ & $4 / 14 / 2007$ & $32.55622 \mathrm{~N} ; 119.45517 \mathrm{E}$ \\
\hline & Jinhua, Zhejiang, China & ZJJH & $3 / 23 / 2007$ & $28.85230 \mathrm{~N} ; 119.46883 \mathrm{E}$ \\
\hline Mountainous region & Weishan, Yunnan, China & YNWS & $7 / 04 / 2007$ & $25.16897 \mathrm{~N} ; 100.14422 \mathrm{E}$ \\
\hline$\left(\mathrm{n}_{\text {ITS1-ITS2 }}=4, \mathrm{n}_{16 \mathrm{~S}}=4\right)$ & Meishan, Sichuan, China & SCMS & $4 / 27 / 2007$ & $30.18314 \mathrm{~N} ; 103.73853 \mathrm{E}$ \\
\hline Littoral hilly region & Fuqing, Fujian, China & FJFQ & $3 / 22 / 2007$ & $25.40488 \mathrm{~N} ; 119.64710 \mathrm{E}$ \\
\hline$\left(\mathrm{n}_{\mathrm{ITS} 1-\mathrm{ITS} 2}=3, \mathrm{n}_{16 \mathrm{~S}}=2\right)$ & Changle, Fujian, China & FJCL & $8 / 26 / 2007$ & $25.70914 \mathrm{~N} ; 119.58699 \mathrm{E}$ \\
\hline Karst landscape region & Yizhou, Guangxi, China & GXYZ & $9 / 04 / 2007$ & $24.21169 \mathrm{~N} ; 108.50488 \mathrm{E}$ \\
\hline$\left(\mathrm{n}_{\mathrm{ITS} 1-\mathrm{ITS} 2}=5, \mathrm{n}_{16 \mathrm{~S}}=3\right)$ & Jingxi, Guangxi, China & GXJX & $9 / 26 / 2007$ & $23.36546 \mathrm{~N} ; 106.29970 \mathrm{E}$ \\
\hline
\end{tabular}

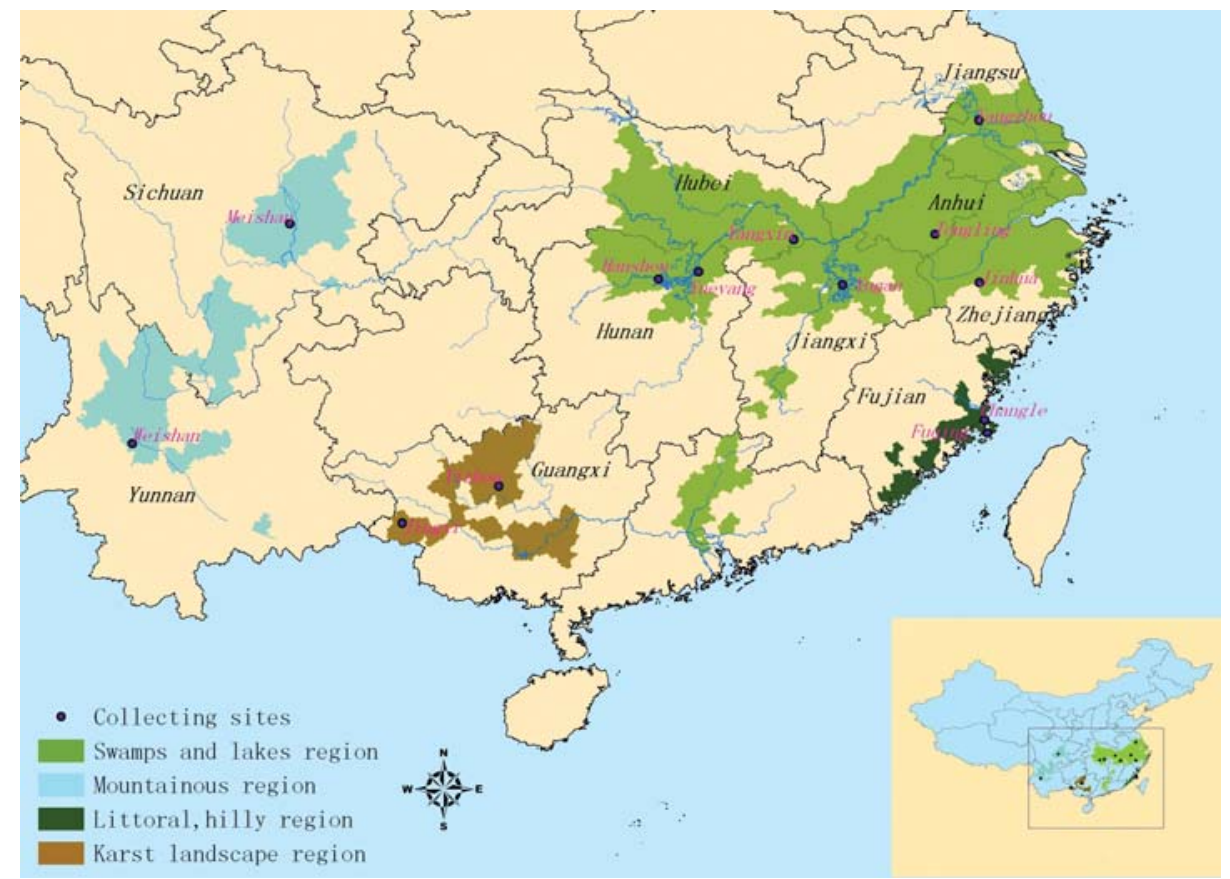

Fig. 1. Distribution of snail landscape ecology populations and collecting sites in schistosome-endemic areas of China. 
extraction procedure (Parayre et al., 2007) using Easy-DNA Kit (QIAGEN GmbH, Hilden, Germany).

\section{DNA amplification and sequencing}

The 5.8S rRNA gene, flanked by the two ITS fragments, was extracted, together with the $16 \mathrm{~S}$ gene sequence, from the 30 snail isolates collected from the four ecological populations. At least four specimens were sequenced from each population. The gene sequences were amplified with a C1000 Thermal Cycler (Bio-Rad, Hercules, CA, USA), using the polymerase chain reaction (PCR) as follows: (i) an initial denaturation at $94^{\circ} \mathrm{C}$ for $3 \mathrm{~min}$; (ii) 35 cycles of $94^{\circ} \mathrm{C}$ for $30 \mathrm{sec}$, annealing at $52^{\circ} \mathrm{C}$ (ITS1-5.8S-ITS2) or at $56^{\circ} \mathrm{C}(16 \mathrm{~S})$ for $30 \mathrm{sec}$ and $72^{\circ} \mathrm{C}$ for $45 \mathrm{sec}$; and (iii) final extension at $72^{\circ} \mathrm{C}$ for $10 \mathrm{~min}$. The primers applied were 16Sar (5'-CGCCTGTTTATCAAAAACAT-3') and 16Sbr (5'- CCGGTCTGAACTCAGATCACGT-3') to amplify a 515 bp region of the $16 \mathrm{~S}$ gene (Attwood et al., 2004), while P1158SF (5'-GAGGATGGATTGGGTTCTCAAGTGT-3') and P1258SR (5'-CTGCGTTAGAAAGGCATGTGGGTTT-3') were applied for the 5.8S rRNA gene together with the two ITS fragments using a design based on an alignment of the U93222 and U93220 sequences of O. hupensis formosana, deposited in GenBank by Lo CT (1997). The resulting PCR products were purified by electrophoresis in $1 \%$ agarose and the bands cut out of the gel with a QIAQuick PCR purification kit (QIAGEN GmbH, Hilden, Germany). The sequences were identified by an ABI 377 automated sequencer (Shanghai Sangon Co. Ltd, Shanghai, China).

\section{Genetic differentiation analysis}

The DNA sequences were assembled and edited using Chromas version 2.3 and BioEdit 7.0.1 (Hall, 1999). The O. hupensis quadrasi sequences were used as the out-group. All sequence datasets were aligned using Clustal X v1.83 (Thompson et al., 1997) and manually checked for accuracy. The analysis of the nucleotide differentiation and the calculation of the genetic distances between the different populations were carried out according to substitution models of the Kimura 2-parameter in MEGA version 4 (Tamura et al., 2007) for closely related isolates. The phylogenetic relationships were analyzed using the methods of maximum parsimony (MP), minimum evolution (ME) and neighbourjoining (NJ) algorithms according to Saitou et al. (1989). Statistical support for the inferred nodes was obtained by bootstrapping in MEGA version 4.0 (Tamura et al., 2007). All bootstrap values were based on the performance of 1000 replicates for the NJ, MP and ME trees.

\section{Spatial correlation analysis}

All coordinates from the sites where the O. hupensis snails were collected were recorded and labeled using Arc View GIS software (ESRI Corp, Redlands, CA, USA) to visualize the geographic distribution map of the O. hupensis collected sites. The geographical distances between collected sites were measured using the mapped data followed by correlation analysis, comparing the spatial and genetic distances for each O. hupensis isolate based on Microsoft Excel (2003). The F-test, the regression coefficient $(\mathrm{R})$ and the decision coefficient $\left(\mathrm{R}^{2}\right)$ between isolates were calculated using SPSS11 (Statistical Products \& Service Solutions) package for Windows (SPSS Inc., Chicago, USA).

\section{Results}

Genetic distance based on the ITS1-5.8S-ITS2 sequence

The various ITS1-5.8S-ITS2 sequences from the 30 individual isolates, representing the four ecological populations, ranged from 752 to 796 bp and showed guanine-cytosine (GC) contents from $49.1 \%$ to $50.3 \%$, respectively (Table 2 ). The alignment of the ITS1-5.8S-ITS2 sequences generated 826 characters with $13.21 \%$ (109) polymorphic 
sites, of which $78.0 \%(85)$ were informative with respect to parsimony. The remaining $22.0 \%$ (24) were singletons and 18 haplotypes were found among them. The 5.8S rRNA gene was located between site 384 and site 521 in the alignment sequence. Only three polymorphic sites were found: a guanine/adenine (G/A) mutation at site 424 (in one isolate from Yangzhou city), a thymine/cytosine (T/C) mutation at site 502 (in five isolates from Guangxi) and a G/A mutation at site 514 (in three isolates from Zhejiang). Most of the polymorphic sites were found in the ITS1 and ITS2 fragments where they formed two "hot spots" at the 160-378 region and the 587-729 region, respectively.

The intra-population genetic distances across the ITS1-5.8S-ITS2 sequence ranged from 0.000-0.011 (in the Karst population from Guangxi) to 0.0000.020 (in the population from the swamps and lakes region). The divergence between the populations was calculated from the inter-population sequences divergence matrix. The genetic distances ranged from 0.0098 between Fujian and the swamps and lakes region to 0.0536 between high-altitude regions (Yunnan and the Karst region of Guangxi) with an average genetic distance of 0.0236 . The population from the mountainous region (Yunnan and Sichuan) was found to be the most divergent one. It differed from all the other populations by a genetic distance ranging from 0.0329 to 0.0536 (Fig. 2).

Genetic distance based on the 165 sequence fragment

The length of the $16 \mathrm{~S}$ rRNA mitochondrial gene from 24 individual isolates (the other six isolates failed the DNA sequencing attempts) from four landscape ecological populations ranged from 505 to $508 \mathrm{bp}$ with the corresponding GC content varying from $33.5 \%$ to $35.1 \%$ (Table 2 ). At the $16 \mathrm{~S}$ locus, with 511 characters generated from the alignment sequences, 14 haplotypes were found. However, only $8.6 \%$ (50) of the sites were polymorphic and $54.0 \%$ (27) of these were informative with respect to parsimony, while the others were single- tons. All the polymorphic sites were scattered in the sequences without any marked regions of concentration.

Intra-population variation was detected in all populations with the greatest seen in the mountainous population from the Yunnan and Sichuan provinces, which showed a maximum genetic distance of 0.0392 between individual isolates. The least genetic distances were seen in the population from the swamps and lakes region (0.000-0.006). When the divergence between the study populations was calculated, the least distance $(0.004)$ was seen between the populations from Fujian and that of the swamps and lakes region (Anhui, Hunan, Jiangxi and Zhejiang). The largest distance $(0.0478)$ was observed between an isolate from Sichuan and an isolate from Guangxi (Fig. 3).

\section{Phylogenetic analysis}

Because the ITS1 and ITS2 sequences, in contrast to the $5.8 \mathrm{~S}$ gene fragment, were found to include a large number of polymorphic sites, the latter sequence was removed. The two ITS fragments were subsequently combined and subjected to phylogenetic analysis with the aim of finding the phylogenetic relationships between the populations from the four geographical areas. The O. hupensis quadrasi was sequenced as an outgroup for the phylogeny analysis.

When the datasets were combined, three substitution models (the maximum composite likelihood, the

Table 2. Statistics related to each locus used in the phylogenetic analyses.

\begin{tabular}{|c|c|c|}
\hline Index & ITS1-5.8S-ITS2 & $16 \mathrm{~S}$ \\
\hline Length $(b p)^{*}$ & $752-796$ & $505-508$ \\
\hline GC content $(\%)$ & $49.1-50.3$ & 34.2 \\
\hline Haplotypes (no.) & 18 & 14 \\
\hline Sites $(b p) * *$ & 825 & 511 \\
\hline Polym. sites $(b p) * * *$ & $109(13.2 \%)$ & $50(9.8 \%)$ \\
\hline PS $(b p)^{* * * *}$ & $85(78 \%)$ & $27(54 \%)$ \\
\hline
\end{tabular}

*Length of amplicon discounting primer sequences; * total number of sites excluding those with alignment gaps; ***number of polymorphic sites; ****number of polymorphic sites with parsimony informative sites within parentheses. 
Jukes-Cantor and the Kimura-2-parameter) were applied to generate the phylogenetic tree according to Tamura et al. (2007). The MP, ME and NJ algorithms resulted in the same phylogenetic topology trees with the level of support being greater for all nodes on the NJ tree (Fig. 2). In comparison to all other populations, three main distinct clades in the phylogenetic trees stood out, i.e. (i) the population from the mountainous regions (Yunnan and
Sichuan); (ii) the population from the Guangxi Karst region; and (iii) the population from the swamps and lakes regions (which included the six provinces mentioned before), including the Fujian population. In this third clade, the Zhejiang isolates were clearly clustered together with Fujian isolates forming a mixed small branch on the phylogenetic tree.

For the $16 \mathrm{~S}$ datasets, the main topology of the phylogenetic trees were equal to those generated

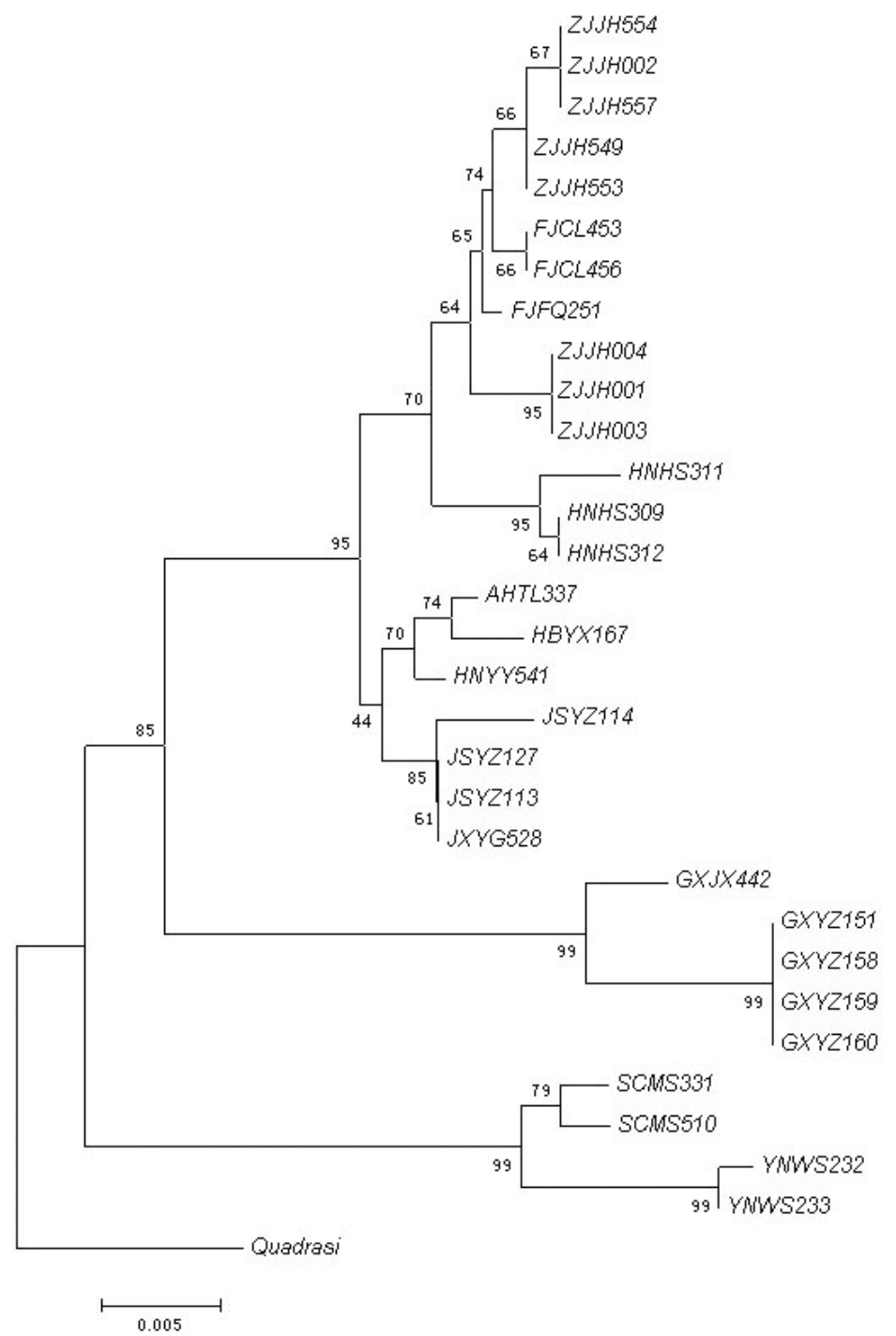

Fig. 2. Phylogeny of the O. hupensis populations based on the combined ITS1 and ITS2 sequences. The tree was generated by neighbour-joining (NJ) analysis, and bootstrap proportions were shown at nodes. Site code was followed by the sample serial number. 


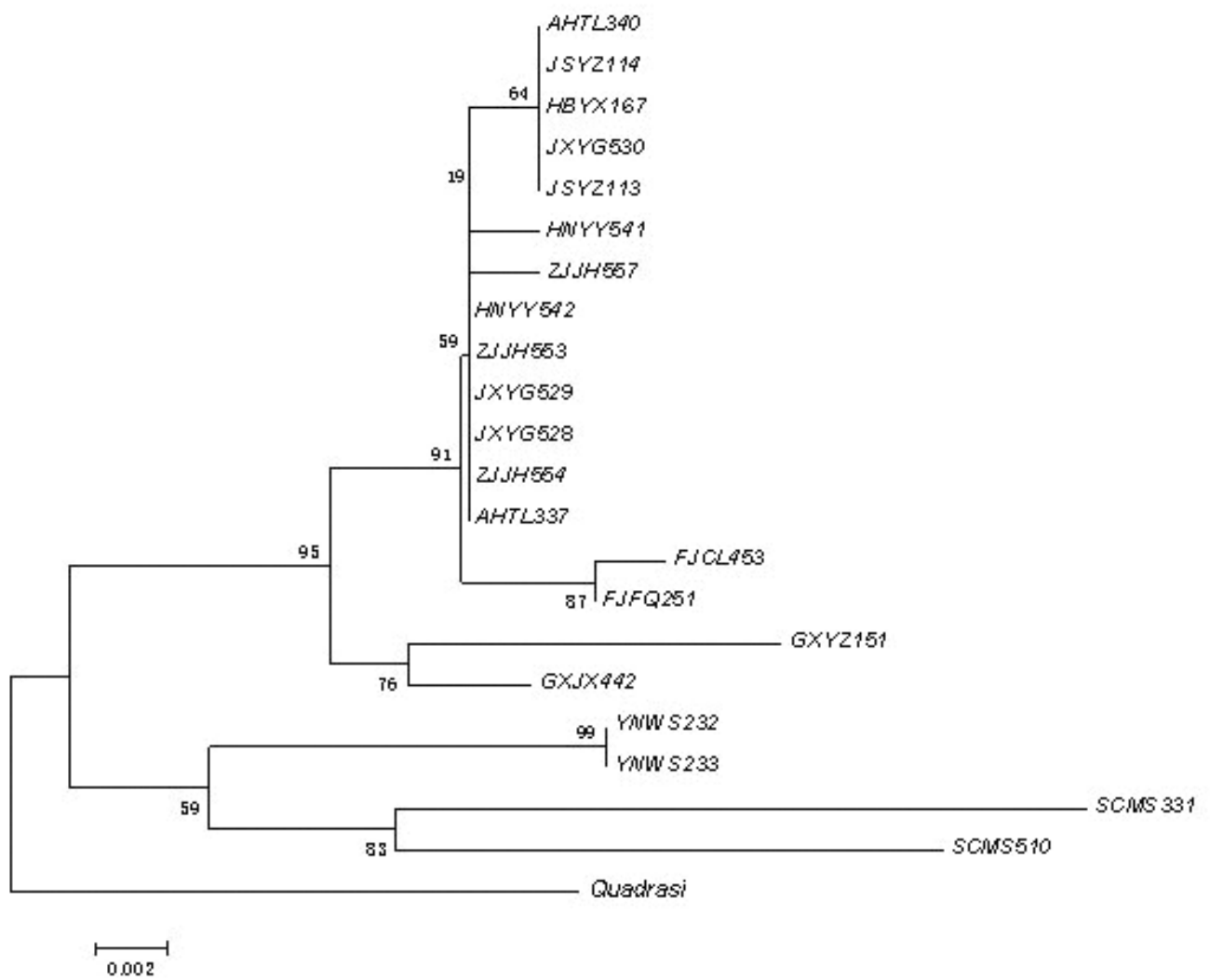

Fig. 3. Phylogeny of the O. hupensis populations based on $16 \mathrm{~S}$ sequences. The tree was generated by minimal evolution (ME) analysis, and bootstrap proportions were shown at nodes. Site code was followed by the sample serial number.

a)

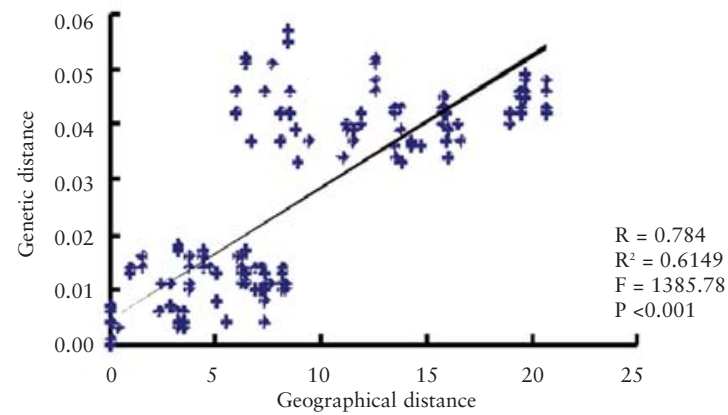

b)

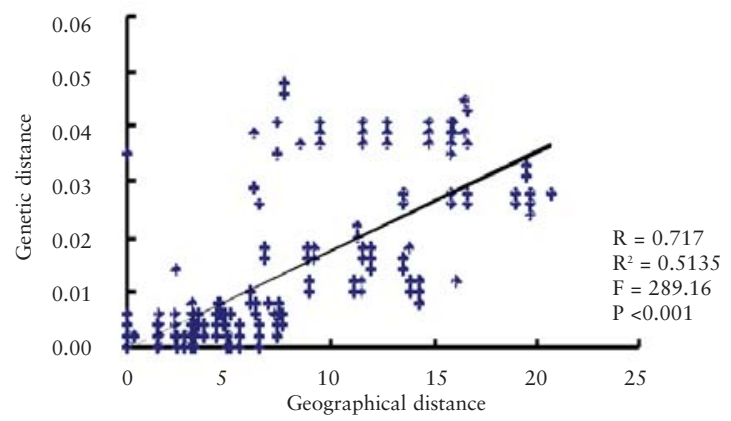

Fig. 4. Correlograms between genetic distance and geographical distance of ITS1-ITS2 and 16S of O. hupensis: a) ITS1ITS2, b) $16 \mathrm{~S}$. 
from the combined ITS1 and ITS2 datasets regardless which method (NJ or ME) was chosen from the Jukes-Cantor, maximum composite likelihood or Kimura-2-parameter models (Fig. 3). Three main clades were observed but the Fujian branch in the third clade was clearly separated from the closed population from the swamps and lakes regions.

\section{Spatial correlation analysis}

The geographical isolation of the four different landscape populations from where the 30 O. hupensis isolates were collected is clearly seen on the map (Fig. 1). The geographical distances between these snail populations showed significant correlations with the genetic distances based on the ITS1-ITS2 and 16S molecular markers (P <0.001) (Fig. 4), confirming a correlation between genetic differentiation and geographical distance at the population level $\left(\mathrm{R}_{\text {ITS1-ITS2 }}=0.78, \mathrm{R}_{16 \mathrm{~S}}=0.72\right)$ (Figs. $4 \mathrm{a}$ and $\left.4 \mathrm{~b}\right)$. Moreover, the decision coefficient $\mathrm{R}^{2}$ ranged from $51.4 \%$ to $61.5 \%\left(\mathrm{R}^{2}\right.$ ITS1-ITS2 $\left.=0.61, \mathrm{R}^{2}{ }_{16 \mathrm{~S}}=0.51\right)$ which indicates the degree of genetic differentiation driven by the geographical difference in the equation.

\section{Discussion}

Since O. hupensis is the only intermediate host that can transmit $S$. japonicum from one final host snail to another, it is clearly of practical importance to establish a robust and ecologically meaningful classification and phylogeny. Efforts to explain the heterogeneities in transmission intensity across mainland China as due to subspecies classification remain controversial (Zhou et al., 2006). However, the investigation presented here, which is based on an integrated approach combining landscape ecology with population genetics, makes it possible to understand genetic differentiation as a combined effect of various characteristics where landscape features influences the gene flow in O. hupensis. The following three main questions require further discussion.
First, is the discovered genetic differentiation driven by the ecology? The study of molecular identities has become a fundamental approach for species differentiation and population genetics ( $\mathrm{Ma}$ et al., 2006). They provide a unique perspective on landscape genetics and emphasize computational and biochemical analyses to better understand evolutionary and functional relationships. An important finding in this respect regards the correlation between genetic differentiation and geographical distance at the population level, which clearly illustrates that genetic differentiation can be attributed to differences in ecological features. This is further supported by the finding that the contribution to genetic differentiation in the snail populations by geographical difference was $51 \%$ to $61 \%$ of all causes in the correlative equation $\left(\mathrm{R}^{2}\right)$. The notion of genetic differentiation as a continuous process issuing from a common origin and driven by ecological variation is strengthened by two molecular markers (ITS1-ITS2 and 16S) producing similar results. However, the phylogeny trees show three branches according to the ITS1-ITS2 marker and four branches according to the $16 \mathrm{~S}$ marker, namely (i) the mountainous region population (Sichuan and Yunnan); (ii) the Karst region population of Guangxi; (iii) the swamps and lakes region population; and (iv) the littoral, hilly region of Fujian. The first and second branches were identical according to both markers, while a difference was noted regarding the third branch, i.e. both markers agreed with the existence of a third branch though the $16 \mathrm{~S}$ data-sets indicated that the third branch should be divided into two (a third and a fourth branch). The two latter branches were clearly observed with the 16S data-sets, which differentiated between the two landscape ecological populations from the littoral hilly regions and the swamps and lakes region of the Yangtze River basin, which is supported by previous AFLP results (Zhou et al., 2007b,c) An interesting discovery is that ITS1-ITS2 data-sets integrated the Fujian isolates with the Zhejiang isolates of the swamps and lakes region. Whether or not this is an example of convergent evolution is discussed in the 
last paragraph of this paper.

The second question is whether the Guangxi population is monophyletic. Based on differences in morphological characters, geographical distribution, ecological environment and parasite susceptibility, O. hupensis guangxiensis was considered a monophyletic subspecies (Liu et al., 1981). They put forward the hypothesis that five subspecies exist in mainland China, namely, O. hupensis hupensis, O. hupensis fausti, O. hupensis tangi, O. hupensis guangxiensis and O. hupensis robertsoni (Liu et al., 1981). However, the later, systematic analysis by Davis et al. (1997), based on morphological data, isoenzyme differences and DNA sequence variation, overturned this analysis with respect to O. hupensis fausti, which is now accepted as a smooth-shelled variant of $O$. hupensis hupensis influenced by ecosystem variations and other environmental factors (Yang et al., 2007, 2008; Zhou et al., 2008). In accordance with Liu et al. (1981), Davis et al. (1997) considered O. hupensis guangxiensis to be sufficiently distinct from O. hupensis hupensis to be regarded as a separate subspecies. This has led to the 3-subspecies classification that includes $O$. hupensis tangi and O. hupensis guangxiensis as two additional, but not sufficiently different, uncertain taxa. This classification has been widely accepted in China even though an in-depth study of O. hupensis guangxiensis is lacking (Qian et al., 1994; Iwanaga, 1995; Yang et al., 2008). However, molecular genetic evidence, together with detailed ecological, morphological and geobiological data, support the 4-subspecies classification according to which the distinction of $O$. hupensis guangxiensis from the Guangxi province is accepted even if full molecular evidence is lacking. The Guangxi samples were clustered with those from Fujian (O. hupensis tangi) in the same clade in AFLP analysis (Zhou et al., 2007b,c). However, in the study presented here, the clade of $O$. hupensis guangxiensis population from Guangxi was distinctly separated from the other populations and formed a separate monophyletic clade based on phylogenetic analysis of two molecular sequences.
Finally, the third question is whether there is convergent evolution between $O$. hupensis tangi and other snails. Although there is no evidence that gene introgression has taken place between O. hupensis tangi (or Fujian isolates) and other snails, it was considered a discrete subspecies or uncertain taxa in the classification system (Liu et al., 1981; Davis et al., 1997; Zhou et al., 2007a,b). The phylogenetic analysis based on the combined ITS1 and ITS2 datasets showed that O. bupensis tangi had clustered with O. hupensis hupensis (Zhejiang) with an indistinct geo-boundary between the two landscape ecologica populations in spite of the close geographical distance presented. In contrast, the clades in the $16 \mathrm{~S}$ genetic cluster, O. hupensis tangi were sufficiently different from $O$. hupensis bupensis to support its independence.

ITS1 and ITS2 have a fast evolutionary rate because of their location at a non-coding rDNA region and are therefore widely used for species identification and analysis of genetic relationships (Stothard et al., 1996). The mitochondrial 16S rRNA gene is relatively conserved and thus exhibits less informative sites with regard to parsimony. It was therefore not surprising that our study found the $O$. bupensis tangi subspecies distributed southeast littoral region of Fujian province to be close to O. hupensis hupensis in the Zhejiang province, which shares a similar environment. Consequently, there maybe a convergent evolution or gene change on the ITS1 and ITS2 sequences between O. hupensis tangi and the Zhejiang isolates of O. hupensis bupensis, which resulted in a shorter genetic distance. This could have been caused by the same type of stress from similar environmental features even if the areas are separated by the Cangshan Mountain (Zhou et al., 2007a), which effectively blocks any exchange.

We concluded that based on the molecular phylogenetic trees investigated, and in accordance with classification according to landscape ecology, this study supports the existence of four main branches of $O$. hupensis largely driven by ecological variations. The hypothesis of landscape ecology as the ultimate arbiter is supported by the unclear genetic 
boundary according to the ITS1-ITS2 datasets between the population from the swamps and lakes region along the Yangtze River basin and that of the littoral hilly region (Fujian). This is further strengthened of O. hupensis guangxiensis now being accepted as a discrete O. hupensis subspecies. However, the convergent evolution between $O$. hupensis tangi and Zhejiang isolates of O. hupensis hupensis needs to be investigated further in the future.

\section{Acknowledgements}

The authors wish to thank Dr. Shan Lv, Dr. Qing Fu, YingJun Qian and He-Xiang Liu of the National Institute of Parasitic Diseases, Chinese Center for Disease Control and Prevention for their kind assistance during the preparation of this manuscript including experiments, data handling, writing and comments. And great thanks to staff from nine provinces for their kind assistance in collecting snail samples.

This study was supported by the Chinese National Science Foundation (project no. 30590373), the UNICEF/ UNDP/World Bank/WHO/Special Programme for Research and Training in Tropical Diseases (TDR; grant no. 970990), and the Ministry of Science and Technology (2003DIA6N009，2005DKA21104， 2007BAC03A02), National Project of Important Infectious Diseases (2008ZX10004-011), and the Excellent Youth Fund of National Institute of Parasitic Diseases, Chinese Center for Disease Control and Prevention.

\section{References}

Attwood SW, Uptham ES, Zhang YP, Yang ZQ, Southgate VR, 2004. A DNA-sequence based phylogeny for triculine snails (Gastropoda: Pomatiopsidae: Triculinae), intermediate hosts for Schistosoma (Trematoda: Digenea): phylogeography and the origin of Neotricula. J Zool Lond 262, 47-56. Chen Z, Zhou XN, Yang K, Wang XH, Yao ZQ, Wang TP, Yang GJ, Yang YJ, Zhang SQ, 2007. Strategy formulation for schistosomiasis japonica control in different environmental settings supported by spatial analysis: a case study from China. Geospat Health 1, 223-231.

Chitsulo L, Engels D, Montresor A, Savioli L, 2000. The global status of schistosomiasis and its control. Acta Trop
77, 41-51.

Davis GM, Zhang Y, Guo YH, Spolsky CM, 1995. Population genetics and systematic status of Oncomelania hupensis (Gastropoda: Pomatiopsidae) throughout China. Malacologia 37, 133-156.

Davis GM, Zhang Y, Guo YH, Spolsky CM, 1997. Systematic status of Oncomelania hupensis (Gastropoda: Pomatiopsidae) throughout China. Studia Marina Sinica 39, 89-95.

Hall TA, 1999. BioEdit: a user-friendly biological sequence alignment editor and analysis program for Windows 95/98/NT. Nucleic Acids Res Symp Ser 41, 95-98.

Hope M, McManus DP, 1994. Genetic variation in geographically isolated populations and subspecies of Oncomelania hupensis determined by a PCR-based RFLP method. Acta Trop 57, 75-82.

Iwanaga Y, 1995. Experimental infection of five subspecies of Oncomelania snails with Angiostrongylus cantonensis. Southeast Asian J Trop Med Public Health 26, 767-773.

Kimura M, 1980. A simple method for estimating evolutionary rate of base substitutions through comparative studies of nucleotide sequences. J Mol Evol 16, 111-120.

Liu YY, Lou ZK, Wang YX, Zhang WZ, 1981. Subspecific differentiation of Oncomelania snails. Acta Zoo-taxonomica Sinica 6, 253- 266.

Ma YJ, Li SZ, Xu JN, 2006. Molecular identification and phylogeny of the Maculatus group of Anopheles mosquitoes (Diptera: Culicidae) based on nuclear and mitochondrial DNA sequences. Acta Trop 99, 272-280.

Manel S, Schwartz MK, Luikart G, Taberlet P, 2003. Landscape genetics: combining landscape ecology and population genetics. Trends Ecol Evol 18, 189-197.

Manel S, Segelbacher G, 2009. Perspectives and challenges in landscape genetics. Mol Ecol 18, 1821-1822.

NCBI taxonomy database. http://www.ncbi.nlm.nih.gov /Taxonomy/. (searched on 4 April, 2009).

Parayre S, Falentin H, Madec MN, Sivieri K, Le Dizes AS, Sohier D, Lortal S, 2007. Easy DNA extraction method and optimisation of PCR-temporal temperature gel electrophoresis to identify the predominant high and low GC-content bacteria from dairy products. J Microbiol Methods 69, 431-441. Qian BZ, Song CC, Thomas KK, 1994. Allozyme variation among populations of Oncomelania hupensis hupensis (Gredler). Chin J Parasitic Dis Control 7, 32- 33. 
Saitou N, Imanishi T, 1989. Relative efficiencies of the FitchMargoliash, maximum-parsimony, maximum-likelihood, minimum-evolution, and neighbor-joining methods of phylogenetic tree construction in obtaining the correct tree. Mol Biol Evol 6, 514-525.

Shi CH, Wilke T, Davis GM, Xia MY, Qiu CP, 2002. Population genetics, micro-phylogeography, ecology, and infectivity of Chinese Oncomelania hupensis hupensis (Gastropoda: Rissooidea: Pomatiopsidae) in the Miao River system: is there a relationship to shell sculpture? Malacologia 44, 333-347.

Spolsky CM, Davis GM, Zhang Y, 1996. Sequencing methodology and phylogenetic analysis: cytochrome b gene sequence reveals significant diversity in Chinese populations of Oncomelania (Gastropoda: Pomatiopsidae). Malacologia 38, 213-221.

Stothard JR, Hughes S, Rollinson D, 1996. Variation within the internal transcribed spacer (ITS) of ribosomal DNA genes of intermediate snail hosts within the genus Bulinus (Gastropoda: Planorbidae). Acta Trop 61, 19-29.

Tamura K, Dudley J, Nei M, Kumar S, 2007. MEGA4: molecular evolutionary genetics analysis (MEGA) software version 4.0. Mol Bio Evol 24, 1596-1599.

Thompson JD, Gibson TJ, Plewniak F, Jeanmougin F, Higgins DG, 1997. The CLUSTAL_X windows interface: flexible strategies for multiple sequence alignment aided by quality analysis tools. Nucleic Acids Res 25, 4876-4882.

Wang LD, Chen HG, Guo JG, Zeng XJ, Hong XL, Xiong JJ, Wu XH, Wang XH, Wang LY, Xia G, Hao Y, Chin DP, Zhou $\mathrm{XN}, 2009$. A strategy to control transmission of Schistosoma japonicum in China. N Engl J Med 360, 121-128.

Wang LD, Utzinger J, Zhou XN, 2008. Schistosomiasis control: experiences and lessons from China. Lancet 372, 1793-1795.

Wilke T, Davis GM, Cui EC, Zhou XN, Xiao Peng Z, Yi Z, Spolsky CM, 2000. Oncomelania hupensis (Gastropoda: Rissooidea) in eastern China: molecular phylogeny, population structure, and ecology. Acta Trop 77, 215-227.

Wilke T, Davis GM, Qiu DC, Spear RC, 2006. Extreme mitochondrial sequence diversity in the intermediate schistosomiasis host Oncomelania hupensis robertsoni: another case of ancestral polymorphism? Malacologia 48, 143-157.

$\mathrm{Xu}$ J, Zheng J, 2003. Preliminary study on genetic diversity of smooth-shelled Oncomelania hupensis from mainland of China by random amplified polymorphic DNA technique. J Trop Dis Parasitol 1, 68-71.

Yang GJ, Utzinger J, Sun LP, Hong QB, Vounatsou P, Tanner M, Zhou XN, 2007. Effect of temperature on the development of Schistosoma japonicum within Oncomelania hupensis, and hibernation of O. hupensis. Parasitol Res 100, 695-700.

Yang K, Wang XH, Yang GJ, Wu XH, Qi YL, Li HJ, Zhou $\mathrm{XN}, 2008$. An integrated approach to identify distribution of Oncomelania bupensis, the intermediate host of Schistosoma japonicum, in a mountainous region in China. Int J Parasitol 38, 1007-1016.

Zhou XN, Wang LY, Chen MG, Wu XH, Jiang QW, Chen XY, Zheng J, Utzinger J, 2005. The public health significance and control of schistosomiasis in China-then and now. Acta Trop 96, 97-105.

Zhou XN, Yang GJ, Yang K, Wang XH, Hong QB, Sun LP, Malone JB, Kristensen TK, Bergquist R, Utzinger J, 2008. Potential impact of climate change on schistosomiasis transmission in China. Am J Trop Med Hyg 78, 188-194.

Zhou YB, Jiang QW, Zhao GM, Yuan HC, 2007a. Subspecies differentiation of Oncomelania hupensis from mainland China. Chin J Schisto Control 19, 485-487.

Zhou YB, Yang MX, Zhao GM, Wei JG, Jiang QW, 2007b. Oncomelania hupensis (Gastropoda: Rissooidea), intermediate host of Schistosoma japonicum in China: genetics, molecular phylogeny based on amplified fragment length polymorphisms. Malacologia 49, 367-382.

Zhou YB, Zhao GM, Peng WX, Wei JG, Jiang QW, 2007c. Spatial genetic correlation analyses of Schistosome japonicum intermediate hosts within Oncomelania hupensis (Gastropoda: Rissooidea) from mainland China based on amplified fragment length polymorphisms. Fudan Uni J Med Sci 34, 207-212.

Zhou YB, Zhao GM, Wei JG, Jiang QW, 2006. Study on the genetic diversity among populations of schistosome intermediate hosts within Oncomelania hupensis (Gastropoda: Rissooidea) in mainland China. Chin J Epi 27, 865-870. 